\title{
ANALYZING SQUARE PLATE IN DIAGONAL COMPRESSION USING BELTRAMI-MICHELL METHODOLOGY
}

\author{
DJELLOUL REZINI \\ LCGE Laboratory, USTO - Mohamed Boudiaf University of Oran, Algeria \\ e-mail: redjellou@yahoo.fr \\ ABDELKRIM KHALDi \\ LRTTFC Laboratory, USTO - Mohamed Boudiaf University of Oran, Algeria \\ KOUIDER BOUzID \\ Metallurgy Department, USTO - Mohamed Boudiaf University of Oran, Algeria \\ YAHIA RAHMANI \\ LRTTFC Laboratory, USTO - Mohamed Boudiaf University of Oran, Algeria
}

\begin{abstract}
This paper is concerned with the "Study of photoelasticity and a photoelastic theoretical investigation of the stress distribution in Square Blocks subjected to concentrated diagonal loads", a thesis topic by M.M. Frocht who developed the well-known semi empirical Shear Stress Difference method. Indeed, the use of the Beltrami-Michell methodology remains quick, when complemented by photoelasticity to acquire Dirichlet's conditions. The synergy of both methods is enhanced with the use of the finite difference method. In addition, a finite element analysis has provided results that will be a supplementary reference for validation. The results obtained have been of lower cost than those obtained by Frocht.
\end{abstract}

Keywords: photoelasticity, hybrid method, Beltrami-Michell, square-plate, diagonal compression

\section{Introduction}

The objective of this paper is to present an alternative solution to the stress distribution in square blocks to that presented in Frocht's thesis entitled "Study of photoelasticity and a photoelastic theoretical investigation of the stress distribution in Square Blocks subjected to concentrated diagonal loads" (Frocht, 1931). Furthermore, some features of the stress distribution are examined by both techniques. We will show, moreover, maps of the whole field of each of the stress components individually, which the method by Frocht cannot provide. In addition, the finite element analysis has provided results that will be a supplementary reference for the validation.

Stress analysis is a classical subject in the field of elasticity theory. Exact solutions to practical problems are not easily determined because of the exact incorporation of the associated physical conditions. The usually used displacement approach degrades the order of the approximation, especially when the state of stress is of principal interest. Therefore, in the alternative direct formulation in terms of stress, the tensor stress field is used as the principal unknown variable (Boresi et al., 2011). This technique is associated with the elliptic equation of Beltrami-Michell forming a well-posed Dirichlet's problem which can be partitioned into two parts; the first approach provides a harmonic field function subject to certain boundary conditions, while the second one investigates the behavior of this function in the neighbourhood of the boundary. Actually, a correct treatment of boundary conditions is one of the major obstacles to the reliable solution to practical problems. 
Although, photoelasticity provides an incomplete solution within the field, nevertheless it always gives a complete one on the boundaries (Frocht, 1941). Isoclinic information will not be needed here, since the isochromatics on the boundaries is the sufficient information (Rezini, 1984) and will be thoroughly used for the proposed stress analysis. However, enhanced with the use of Finite Difference Method (FDM), the aim of this paper is to propose a photoelastic-numerical hybrid method for stress analysis within any irregularly shaped domain in plane stress conditions. Thus, the Square Block will be an appropriate application for the validation of the present model.

\section{Elastic plane stress boundary value problem}

The plane stress problem considered is referred to as an elastic planar layer subjected to symmetric in-plane loading in the case of the diagonal compressed Square Block. Given the plane stress assumptions, this problem is firstly governed by the necessary equilibrium conditions. As a result of the forces applied at the boundary, the stress tensor $\boldsymbol{\sigma}$, a symmetric rank two tensor field, satisfies the following equilibrium condition either in the absence or for constant body forces for each $i$

$$
\sum_{j=1}^{2} \frac{\partial \sigma_{i j}}{\partial x_{j}}=0
$$

Moreover, the plane stress problem is defined as the elastic planar layer $\Omega$ on the stress assumption that $\left(\sigma_{33}=\sigma_{13}=\sigma_{23}=0\right) ;\left(x_{1}, x_{2}, x_{3}\right) \in \Omega$. Furthermore, the stress field in the middle layer is reasonably described by the three field components $\sigma_{11}\left(x_{1}, x_{2}\right), \sigma_{22}\left(x_{1}, x_{2}\right)$, and $\sigma_{12}\left(x_{1}, x_{2}\right)$ which form the symmetrical stress tensor, of which the trace is expressed as follows

$$
\operatorname{trace}(\boldsymbol{\sigma})=\Sigma_{\sigma}\left(x_{1}, x_{2}\right)=\sigma_{11}+\sigma_{22}
$$

In terms of stress, the invariant $\Sigma_{\sigma}\left(x_{1}, x_{2}\right)$ has the fundamental properties of a harmonic function that satisfies the Laplace-potential equation. In fact, if the Laplace operator is applied to the trace of the stress tensor given in (2.2), the well-known Laplace potential equation for the stress sum is obtained

$$
\sum_{j=1}^{2} \frac{\partial^{2} \Sigma_{\sigma}\left(x_{1}, x_{2}\right)}{\partial x_{j}^{2}}=0
$$

Equation (2.3) as stated is the compatibility condition in terms of stress for planar version. When equilibrium equations (2.1) are derived in a suitable form and subtracted from each other, the following equality in terms of the second order derivatives will result

$$
\frac{\partial^{2} \sigma_{11}}{\partial x_{1}^{2}}=\frac{\partial^{2} \sigma_{22}}{\partial x_{2}^{2}}
$$

In a similar way, by adding both equations, the determining Poisson equation for the shear stress is obtained, and is given as follows

$$
\Delta \sigma_{12}=-\frac{\partial^{2}\left(\sigma_{11}+\sigma_{22}\right)}{\partial x_{1} \partial x_{2}}
$$

where $\Delta$ is referred to as the two-dimensional Laplace operator. Laplace's potential equation (2.3) may be rewritten in the extended form shown below

$$
\Delta \sigma_{11}=-\frac{\partial^{2} \sigma_{22}}{\partial x_{1}^{2}}-\frac{\partial^{2} \sigma_{22}}{\partial x_{2}^{2}}
$$


The incommoding member in equation (2.6) will be replaced by its equivalent one given in equation (2.4), so that an equation of the Poisson type for the normal stress component is obtained

$$
\Delta \sigma_{11}=-\frac{\partial^{2}\left(\sigma_{11}+\sigma_{22}\right)}{\partial x_{1}^{2}}
$$

In a cyclic permutation, the equation for the normal stress in the other direction is established likewise

$$
\Delta \sigma_{22}=-\frac{\partial^{2}\left(\sigma_{11}+\sigma_{22}\right)}{\partial x_{2}^{2}}
$$

Equations (2.5), (2.7) and (2.8) are the well-known Beltrami-Michell equations; with the requirement that the potential equation solution of the sum of stresses given in (2.3) at the stress field must be available. Other formulations shown in the literature may lead to the same equations (Hetnarski and Ignaczak, 2011). Furthermore, it is essential, however, to notice the fact that the Beltrami-Michell equations are independent of elastic constants for the two-dimensional case (Muskhelishvili, 1975).

\section{Photoelasticity treatment of the problem}

The photoelastic experiment (Ramesh, 2008; Frocht, 1941, 1948) provides only two experimental parameters for each point: the isochromatic fringe order $N$ which is proportional to $\left(\sigma_{1}-\sigma_{2}\right)$ and $\theta$, the isoclinic parameter. However, three characteristics are needed to fully define the stress state. Photoelasticity provides therefore an incomplete solution as is symbolically sketched below

$$
\left(\sigma_{11}, \sigma_{22}, \sigma_{12}\right) \Longleftrightarrow \begin{cases}N & \text { isochromatic order } \\ \theta & \text { isoclinic parameter }\end{cases}
$$

Both the two-dimensional basic equation of photoelasticity and the procedure are well documented in the literature (Sharafutdinov, 2012). Photoelasticity and the SSD-method form a single semi empirical hybrid method which calculates stress components within a grid starting from initial values at the boundary points. The SSD-method is based on the step-by-step integration of one of the differential equations of equilibrium. In a Cartesian $(x, y)$ plane, for any particular $y$-ordinate, equation (2.1) can be integrated in the $x$-direction

$$
\sigma_{x}\left(x_{1}\right)=\sigma_{x}\left(x_{0}\right)-\int_{x_{0}}^{x_{1}} \frac{\partial \tau_{x y}}{\partial y} d x
$$

where the partial derivative $\partial \tau_{x y} / \partial y$ is a function of the $x$-coordinate. In practice, $\tau_{x y}$ is determined by photoelasticity from the isochromatic fringe order $N$ and the isoclinic parameter $\theta$ viewed in the $(x, y)$-plane (Fig. 1) using the following photoelasticity law

$$
\tau_{x y}=\frac{N f}{2 t} \sin 2 \theta
$$

where $f$ and $t$ are the material constant and thickness of the model, respectively. When the loaded Square Block model is viewed in the polariscope, a fringe pattern called isochromatic is apparent.

The Square Block photoelastic data are shown in Fig. 1 where the stress patterns are on the right-hand side, and the corresponding isoclinics are on the left-hand side. The stress separation 


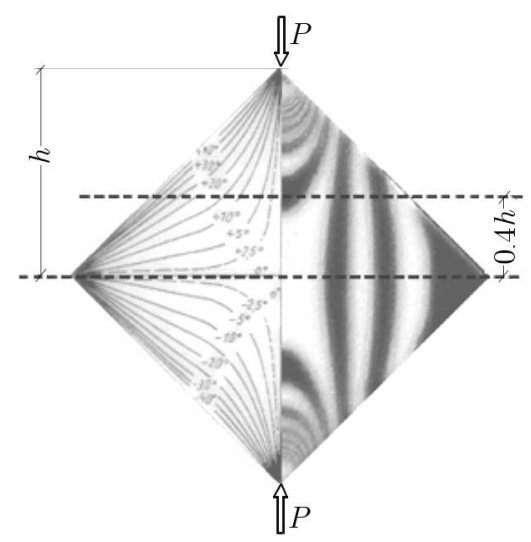

Fig. 1. Isoclinics and isochromatic fringes data of the diagonally compressed Square Block

will be carried out along the straight line, indicated by $X$. Along the horizontal line $y=$ const, for the SSD-method, the central-difference between 2 auxiliary lines parallel to $y$ at a distance of $\Delta y$, the partial differential quotient of the shear stress is approximated as follows

$$
\frac{\partial \tau_{x y}}{\partial y} \cong \frac{\Delta \tau_{x y}}{\Delta y}
$$

Substituting expression (3.3) into equation (3.1) and using elementary summation along the grid nodes in the photoelasticity law, equation (3.2) leads to the following separated stresses both in the $x$ and $y$-directions

$$
\left.\sigma_{y}\right|_{i}=\left.\sigma_{y}\right|_{0}+\left.\sum_{k=1}^{i}\left(\frac{\Delta \tau}{\Delta x} \Delta y\right)_{k} \quad \sigma_{x}\right|_{i}=\left.\sigma_{x}\right|_{0}+\sum_{k=1}^{i}\left(\frac{\Delta \tau}{\Delta y} \Delta x\right)_{k}
$$

It is shown that even if the error introduced by the measurement of the isochromatic parameter may not be important, the error due to the isoclinic (stress direction) measurement can be very significant (Kuske, 1959, 1971). Since the SSD-method involves step-by-step integration, the error introduced will be cumulative.

Concerning the boundary values, the state of stress is most evident on the free boundaries. At each point of the free boundary either $\sigma_{1}$ or $\sigma_{2}$ vanishes; the stress fringe pattern gives immediately the numerical value of the remaining principal stress. In addition, the sign of the boundary stress can be determined by considering the external loading or by "nail" pressure on the boundary (Kuske and Robertson, 1974). The required boundary potentials arise automatically from photoelastic study of the specimen in which the principal stress sum can be obtained readily because they are identical to the principal stress difference on the free boundary (Mangal and Ramesh, 1999; Petrucci, 1997; Pinit and Umezaki, 2007), from which the equality of their absolute values at the boundary is deduced

$$
\sigma_{B}=\left|\left(\sigma_{1}+\sigma_{2}\right)_{\partial \Omega}\right|=\left|\left(\sigma_{1}-\sigma_{2}\right)_{\partial \Omega}\right|
$$

Besides, the harmonic state of the stress invariant satisfies Laplace equation (2.3); and it is usually used to determine the interior potential of the stress sum with the necessary knowledge of boundary conditions (Fernàndez et al., 2010). The accuracy of the boundary values of the stress sum is absolutely decisive for the results. This is true because the stress sum occurs each time as a source function of the set of the Beltrami-Michell equations. According to the law of photoelasticity, the stress sum is proportional to the fringe order on the free boundaries

$$
\left.\left(\sigma_{x}+\sigma_{y}\right)\right|_{\partial \Omega}=\left.\kappa N(x, y)\right|_{\partial \Omega}
$$


Knowing the inclination angle $\theta$ (or the slope) at a given point on the model edge and the tangential stress $\sigma_{B}=\kappa N(x, y)$ at the same point, the Cartesian stresses components are easily computed using separately the equilibrium conditions as follows

$$
\sigma_{x}=\sigma_{B} \cos ^{2} \theta \quad \sigma_{y}=\sigma_{B} \sin ^{2} \theta \quad \tau_{x y}=-\sigma_{B} \sin \theta \cos \theta
$$

Commonly, only one black-and-white photograph of the boundary-isochromatic fringe patterns of the model for the input data is needed. The simplest way of fringe data recording is to use a digitizing tablet and to "click" (pick up) on the centre of each fringe at a density that adequately represents the fringe centreline (such as Gauss distribution) on the breadth (Rezini, 1984). This simple acquisition technique has the significant advantage of allowing the user maximum control of the number and location data. Per boundary, the coordinates of the boundary points are recorded along with their isochromatic values and tabulated in an array $\left(x_{i}, y_{i}, N_{i}\right)$, where $i=1, \ldots, n$ labelling each recorded point in this array. It is also all of the entire work necessary for the data input procedure. It is to be pointed out that the matrices of each system of the finite difference algebraic equations will be automatically occupied, taking into account the recorded boundary values, as described above. In the next step, a processing subprogram (solver) is started in order to perform the numerical analysis. An output program shall be provided.

\section{Finite difference implementation on the Square Block}

Due to its definition, the FDM is the most important and certainly still dominant numerical method in the partial differential equations modelling. In order to approximate the solution of a partial differential equation by its nodal values at a set of grid points, the FDM often requires the mesh points to be uniformly placed, so that the derivatives of the unknown function at grid points can be accurately approximated. Difficulties principally occur in the FDM discretization of the homogeneous and mixed derivatives at irregular shaped boundaries. To overcome this restriction and in order to solve partial differential equations that are defined on domains with irregular geometry, numerous variants are proposed in several works (Collatz, 1960; McKenney et al., 1996). Many procedured exist on this subject; e.g. McKenney et al. (1996) propose a fast Poisson solver for complex geometries. In this way, Zhang (1998) developed a multigrid solution to Poisson's equation. In the present implementation, a flexible 5-point stencil is systematically used for the discretization of the homogeneous and the mixed partial derivatives occurring in both Laplace equation (2.3) and in the right-hand side of Poisson equations (2.5), (2.7) and (2.8).
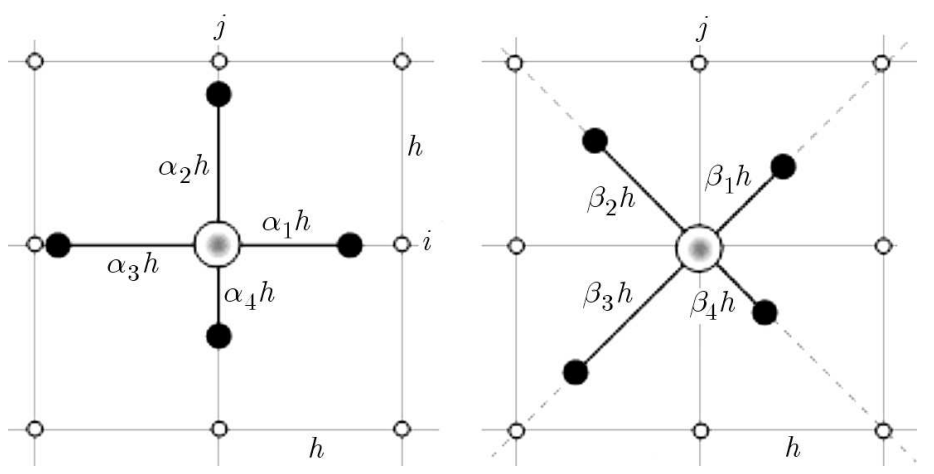

Fig. 2. Unequal armed difference star: (a) normal grid, (b) skewed grid

Consider the general case of a group of five points, of which spacing is non-uniform, arranged in an unequal-armed star. We represent each distance by $\alpha_{i} h$, where $\alpha_{i}$ represent the respective 
fractions of the standard spacing $h$ from the discretization central point as shown in Fig. 2a. Based on Taylor's theorem, the derivative of a function at a point can be approximated by the linear combination of function values at nearby points including the function itself (Dahlquist and Bjorck, 1974; Smith, 1985). The definition of difference quotients and of the discrete Laplace operator also has to be modified. This leads to the Shortley-Weller approximation (Shortley and Weller, 1938)

$$
\left.\Delta \phi\right|_{h}=C_{0}^{+} \Phi_{0}+C_{1}^{+} \Phi_{1}+C_{2}^{+} \Phi_{2}+C_{3}^{+} \Phi_{3}+C_{6}^{+} 4 \Phi_{4}= \begin{cases}0 & \text { Laplace } \\ \left.f\right|_{k=0} & \text { Poisson }\end{cases}
$$

In this symbolic equation, (4.1), the coefficients of the finite difference equations are defined depending on the spacing to the boundaries. The Shortley-Weller coefficients for the discrete Laplace operator $\left.\Delta\right|_{h}$ will be distinctly expressed as follows

$$
\begin{aligned}
C_{1}^{+} & =\frac{1}{h^{2} \alpha_{1}\left(\alpha_{1}+\alpha_{3}\right)} & C_{2}^{+} & =\frac{1}{h^{2} \alpha_{2}\left(\alpha_{2}+\alpha_{4}\right)} \\
C_{3}^{+} & =\frac{1}{h^{2} \alpha_{3}\left(\alpha_{3}+\alpha_{1}\right)} & C_{4}^{+} & =\frac{1}{h^{2} \alpha_{4}\left(\alpha_{4}+\alpha_{2}\right)} \\
C_{0}^{+} & =-\sum_{k=1}^{4} C_{k}^{+} & &
\end{aligned}
$$

The motivation of the following approach is to produce an accurate method for treating mixed derivatives. Near the boundary, the approximation of the second order mixed derivatives requires a 9-point stencil. This task is not easy to handle with respect to the aimed "automatization" of the method. A transformation by rotation $45^{\circ}$ of the grid (stencil) to obtain a 5-point formula is also suggested. Notice that the 2-dimensional Taylor series approach, according to the approximation of mixed derivatives, leads to a similar form of Shortley-Weller's coefficients in a $45^{\circ}$ skewed stencil as shown in Fig. $2 \mathrm{~b}$

$$
\left.\frac{\partial^{2} \phi}{\partial x \partial y}\right|_{h}=C_{0}^{\times} \Phi_{0}+C_{1}^{\times} \Phi_{1}+C_{2}^{\times} \Phi_{2}+C_{3}^{\times} \Phi_{3}+C_{4}^{\times} \Phi_{4}
$$

The finite difference coefficients for mixed derivates (4.3) can be expressed in individual terms as follows

$$
\begin{aligned}
C_{1}^{\times} & =\frac{1}{2 h^{2} \beta_{1}\left(\beta_{1}+\beta_{3}\right)} & C_{2}^{\times} & =\frac{-1}{2 h^{2} \beta_{2}\left(\beta_{2}+\beta_{4}\right)} \\
C_{3}^{\times} & =\frac{1}{2 h^{2} \beta_{3}\left(\beta_{3}+\beta_{1}\right)} & C_{4}^{\times} & =\frac{-1}{2 h^{2} \beta_{4}\left(\beta_{4}+\beta_{2}\right)} \\
C_{0}^{\times} & =-\sum_{k=1}^{4} C_{k}^{\times} & &
\end{aligned}
$$

The above symbols $(+, \times)$ are used for normal and skewed network stencil, respectively. By definition, the irregular 5-point-difference star is present when at least one of its star boughs (arms) becomes smaller than the mesh size $h$. The irregular difference star is the most general case and for the regular ones, the following condition is required $\alpha_{k}=\beta_{k}=1, k \in\{1,2,3,4\}$.

Furthermore, in this study, the direct method which produces an exact solution in a finite number of operations is used (Duff et al., 1986). When solving successively the Laplace and the Poisson systems of equations, the errors in the solutions arise only from the rounding off and truncation errors in the computational calculation (Smith, 1985; Collatz, 1960). This method is tested on several application examples and is found to produce very good results (Rezini, 1984). 


\section{Results of methods and discussion}

Before the PCs had appeared, the SSD-method was done by manual calculations. One imagines the excess of labour to investigate the Square Block problem. However, because we already have the results of Frocht (1948, pp. 265-269), the feasibility of this method will be proved on this application example. Isochromatics (in orders) have been superimposed for convenience over the isoclinic parameters in the field around the straight line $X$, as depicted in Fig. 1 . The stressintegration can be started from the initial values, and the stress-separation will be accomplished according to numerical integrations (3.4), as mentioned previously. The initial stress values are obtained from the boundary values at a point from conditions (3.7). For the stress-separation along the straight line, instead of using relation (3.4), Frocht proceeds as follows

$$
\begin{gathered}
\left.\sigma_{x}\right|_{i}=\left.\sigma_{x}\right|_{0}-\sum_{0}^{i} \Delta \tau_{x y}\left(\frac{\Delta x}{\Delta y}\right) \\
\sigma_{y}=\sigma_{x} \pm \sqrt{\left(\sigma_{1}-\sigma_{2}\right)^{2}-4 \tau_{x y}^{2}}
\end{gathered}
$$

The plus or minus signs in front of the radical in (5.1) $)_{1}$ correspond to the two possible positions of the centre of Mohr's circle. Accordingly, $\sigma_{1}$ lies furthermost on the right and $\sigma_{2}$, being the furthermost, on the left of the circle.

The initial value of $\left.\sigma_{x}\right|_{0}$ for the step-by-step integration is evaluated from the boundary value of $q_{0}$ (fringe order negative) and $\theta_{0}$, which are 0.80 fringe and $45^{\circ}$, respectively. The initial stresses in both the $x$ direction and the $y$ direction get the following respective values

$$
\left.\sigma_{x}\right|_{0}=q_{0} \cos ^{2} 45^{\circ}=-0.8 \cdot 0.5=-0.40 \text { fringe }
$$

and

$$
\left.\sigma_{y}\right|_{0}=q_{0} \sin ^{2} 45^{\circ}=-0.8 \cdot 0.5=-0.40 \text { fringe }
$$

The initial shear stress value becomes

$$
\left.\tau_{x y}\right|_{0}=-q_{0} \sin 45^{\circ} \cos 45^{\circ}=-\frac{q_{0}}{2}=+0.8 \cdot 0.5=+0.40 \text { fringe }
$$

In this case, it is important to point out the difference between both procedures: in Frocht's SSD-process, the stress component $\sigma_{y}$ is determined by means of Mohr's circle statement $(5.1)_{2}$; while the isoclinic parameter $\theta$ is used for the integration. The cumulative error in the integration process has also an effect on the stress-separation. It is noted that the determination of $\theta$ isoclinic parameter in its physical range remains, generally, a difficult problem (Fernàndez, 2011). Several works have been published concerning this topic; see for example (Mangal and Ramesh, 1999; Siegmann et al., 2011; Ajovalasit and Zuccarello, 2000). Besides, in the Beltrami-Michell Boundary Value Problem (B-M BVP), each stress component being subject to its values on the boundary form a BVP individually. In addition, it is noted that the Square Block edges direction (slope) is a principal stress direction itself in the case of a free boundary. Otherwise, this shows the long process in achieving the results by means of the SSD-method (see diagram; Fig. 3). Mainly, the fact it is noticed that the separation of stresses is established along a straight line only.

The need for full-field separation of stresses is more advantageous, and this is why B-M BVP is more appropriate. In order to make full comparisons, the same representation as that of Frocht (Fig. 3) is adapted.

In order to re-examine the stress distribution for further validation, a numerical analysis has been carried out using the finite element analysis for the same Square Block loading situation. 


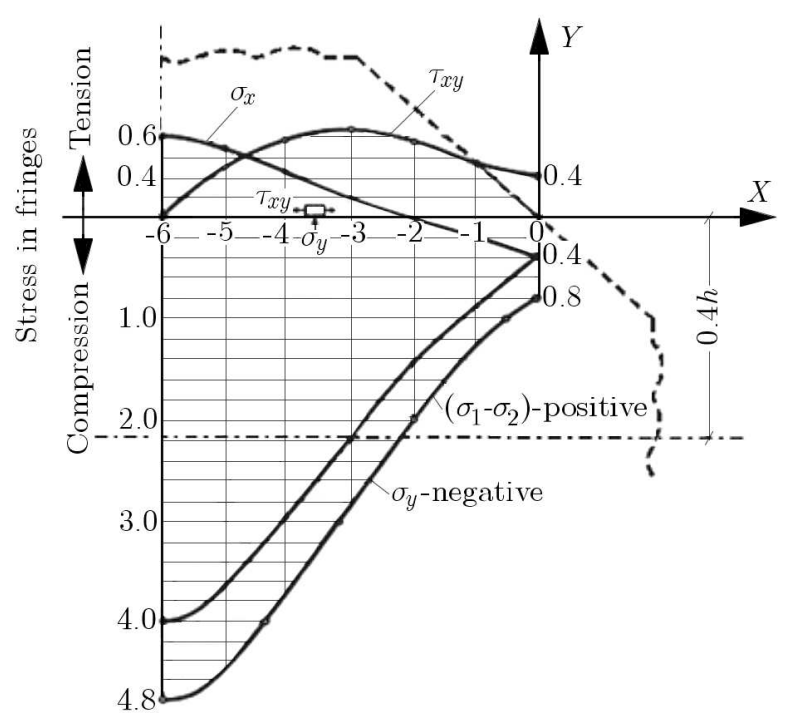

Fig. 3. Cartesian stress components distributions on section $X$ (Frocht, 1948, pp. 265-269)

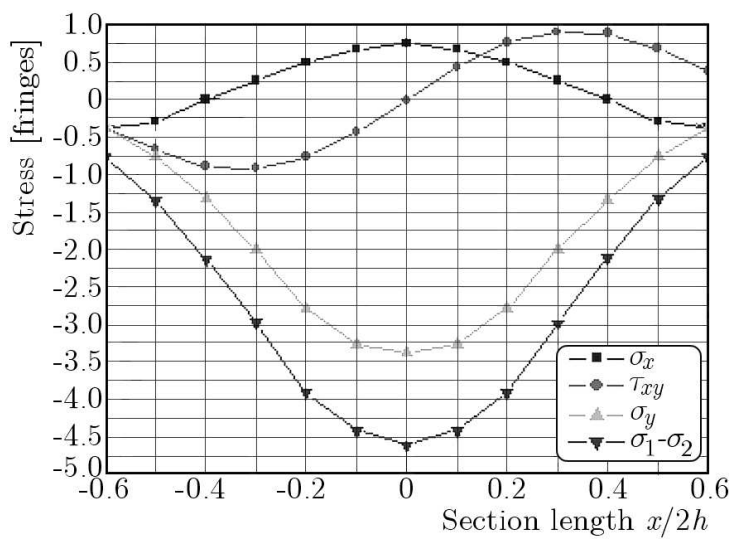

Fig. 4. Cartesian stress component distributions on section $X$ (referring to this work)

The FEA has been performed using ANSYS software. PLANE82 elements have been used to model the Square Block subjected to diagonal compression. This type of element provides more accurate results for mixed (quadrilateral-triangular) automatic meshes and can tolerate irregular shapes without as much loss of accuracy. Such 8-node elements have compatible displacement shapes and are well suited to model curved boundaries (Madenci and Guven, 2005).

After preliminary tests, the meshing configuration shown in Fig. 5 has been opted for; a number of 3491 PLANE82-elements (10720 Nodes) have been used.

The static balance across section $\mathrm{X}$ is then stated when the sum of vertical forces vanishes. This condition of equilibrium in the loading direction provides the necessary (check) verification of each method used, permitting the comparison of the numerical results. All geometrical dimensions and the load necessary for the result verification are shown in Fig. 5. The units are also kept Anglo-American. Furthermore, the stress distributions along the straight line $X$ will be compared with those of the SSD-method and of FEA. Accordingly, the equilibrium across the horizontal section may be re-examined. The normal stress $\sigma_{y}$ acting within each cross section must balance the load $P_{y}$ each time. The area under the $\sigma_{y}$-curve (Fig. 3) has been measured by means of a planimeter and found to be $11.2 \mathrm{in}^{2}$. The length of the half section $0.6 \mathrm{~h}$ has been found to be 4.065 in (Frocht, 1941, pp. 270). Hence

$$
\sigma y=\frac{11.2}{4.065}=2.75 \mathrm{in}=2.2 \text { fringes }
$$




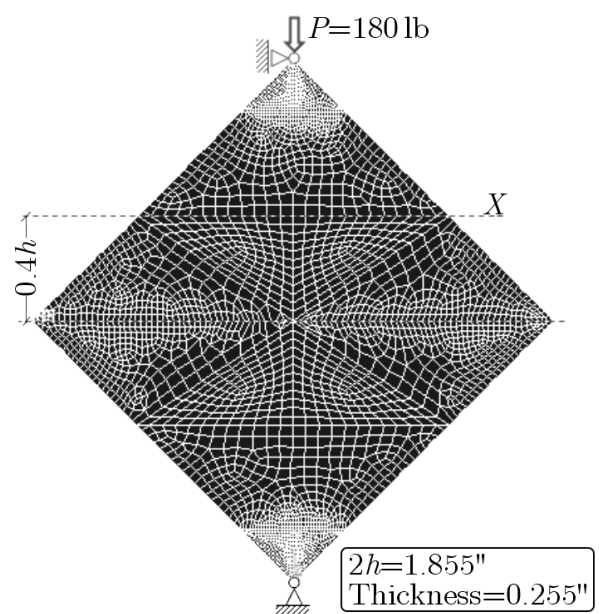

Fig. 5. Meshing configuration of the Square Block and associated boundary conditions

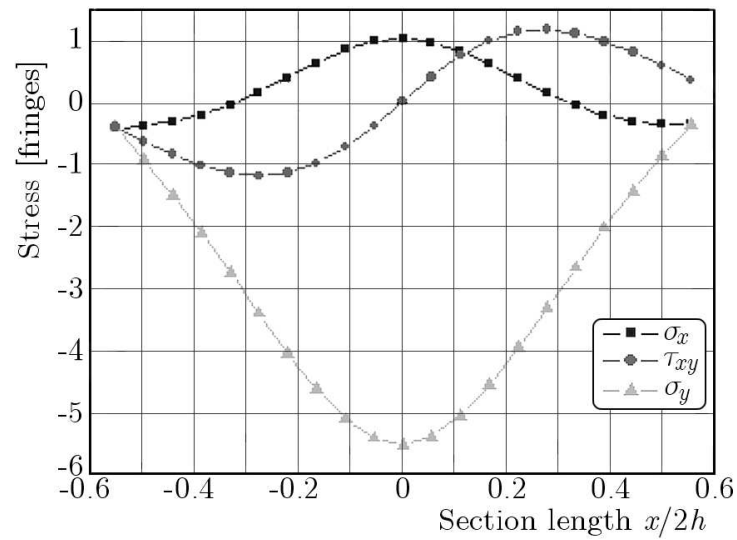

Fig. 6. Cartesian stress component distributions on section $X$ using FEA

Multiplying this value by the model compressive fringe value of $284 \mathrm{psi}$, we have: $\sigma_{y}=2.2 \cdot 284=$ $625 \mathrm{psi}$. The force $P_{y}$ acting on the section of length $0.6 h$ is then given by: $P_{y}=625 \cdot 1.855$. $0.255 \cdot 0.6=177.5 \mathrm{lb}$. This result differs from the applied load of $180 \mathrm{lb}$ approximately by $-1.5 \%$. Concerning our results, the integral of the $\sigma y$-curve (Fig. 6) is calculated. By taking into account our scale, the following result is obtained: $\sigma_{y}=2.18 \cdot 284=619$ psi. Hence, the calculated force resulting from our calculation method is: $P_{y}=619 \cdot 1.855 \cdot 0.255 \cdot 0.6=175.7 \mathrm{lb}$.

Therefore, the error induced is approximately $-2.4 \%$. This discrepancy is not a crucial one. The reasons leading to this error magnitude will be discussed later. In contrast, the FEA results are the most accurate (see Table 1) in this analysis.

Table 1. Comparison of present results with Frocht's and FEA

\begin{tabular}{|l|c|c|}
\hline Result of & $P_{y}[\mathrm{lb}]$ & Error [\%] \\
\hline \hline Frocht & 177.50 & -1.5 \\
\hline FEA & 179.75 & -0.1 \\
\hline this work & 175.70 & -2.4 \\
\hline
\end{tabular}

Referring to the available experimental results, those achieved by the photoelastic-numerical hybrid method are in good agreement with a relative difference of about $-1 \%$. Furthermore, it is worth emphasizing that the occurring small deviations in the results obtained are due to the procedure of the graphical representation rather than to inaccuracy in the calculation. 
Moreover, the component stress distribution is automatically interpolated at the grid points in the neighbourhood of the straight line $X$, which explains once more the deviation of the results. On the one hand, the designated lines along which the results will be compared cannot be identical in the absolute. Moreover, there is a significant difference in terms of the process used to expose stress distribution maps in the reference work (Fig. 4) likewise for FEA (Fig. 6). Separating the result data in individual components usually provides stresses at only discrete locations. For this purpose, the field results are presented in Figs. 7 and 8.

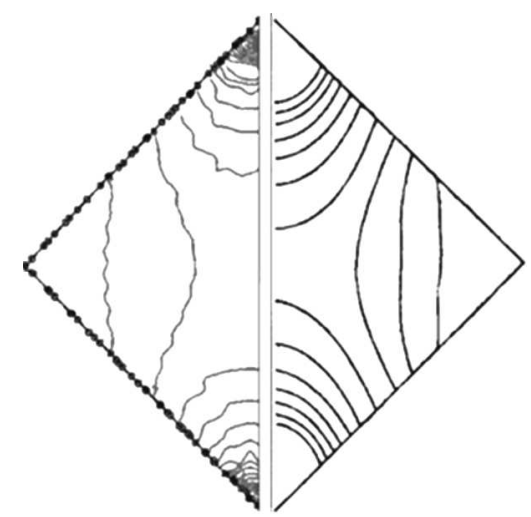

Fig. 7. Isopachic stress patterns; LHS calculated and RHS measured
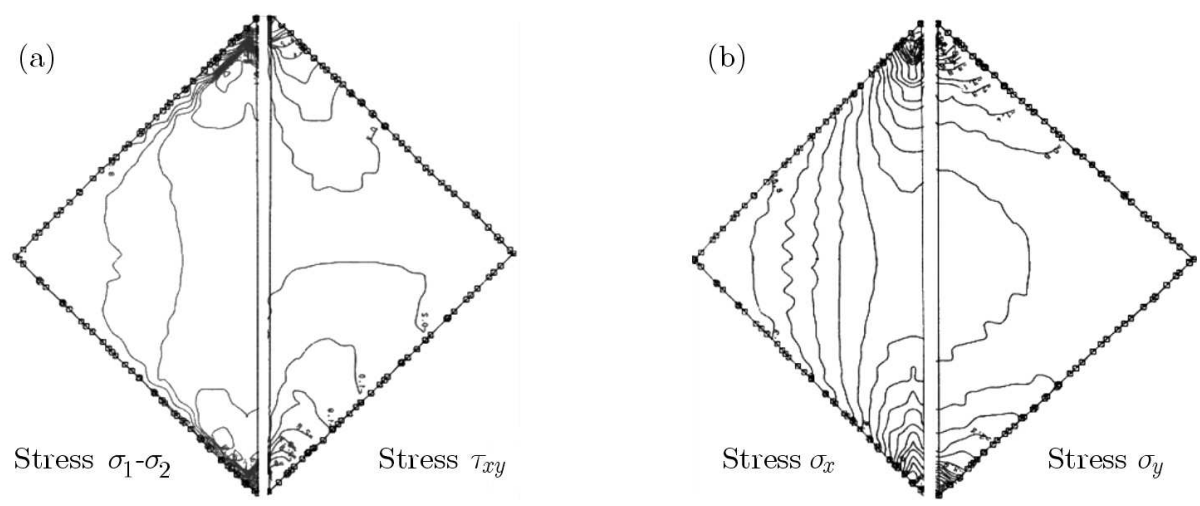

Fig. 8. Full-field distributions of each stress

\section{Conclusion}

In the present study, validation of the stress state results obtained by two comparative methods has been carried out using supplementary FEA numerical models. A very good qualitative agreement between the three methods has been found. As treated by Frocht, the true whole field isoclinic angle and phase difference are the key data to obtain the stress distribution in a birefringent model. The feasibility of this method has been proved by the Square Block experiment under in-plane compression, wherein only boundary isochromatic fringe data are needed. Furthermore, the developed SSD-method by Frocht still provides accurate results. The aim of this paper is to show the efficiency of an additional method for stress-separation without needing field information as in the case of the SSD-method; and to quickly obtain results with an acceptable accuracy. Based on the numerical solving of the B-M BVP of Dirichlet's type, this method reduces the photoelastic data to the minimum, in order to separate the prevailing stresses. Further, the method facilitates the automatic process for generating stress component maps (Ajovalasit and Zuccarello, 1998) and reconstructing the full-field stress tensor using little equipment. In 
regard to large utilization in the experimental-numerical photoelasticity, the next step will be to develop an automatic system that allows the computer to control both the image acquisition of boundary isochromatic fringes and the shape recognition simultaneously. In fact, the present paper has shown that the successful use of a hybrid method can thereby reduce the amount of measurements required, increase the accuracy of numerical results and, at the same time, allow quick determination of the complete stress distribution within the studied part.

\section{References}

1. Ajovalasit A., Zuccarello B., 2000, Limitation of Fourier transform photoelasticity: influence of isoclinics, Experimental Mechanics, 4, 384-392

2. Ajovalasit A., Barone S., Petrucci G., 1998, A review of automated methods for the collection and analysis of photoelastic data, Strain, 33, 75-91

3. Boresi A.P., Chong K.P., Lee J.D., 2011, Elasticity in Engineering Mechanics, 3rd Edit., John Wiley \& Sons, Inc.

4. Collatz L., 1960, The Numerical Treatment of Differential Equations, Springer

5. Dahlquist G., BJorck A., 1974, Numerical Methods, Prentice-Hall, Englewood Cliffs, NJ

6. Duff I., Erisman A., Reid J., 1986, Direct Methods for Sparse Matrices, Oxford University Press, England

7. FERnÀndez M.S.-B., 2011, Towards uncertainty evaluation in photoelastic measurements, Journal of Strain Analysis, 45, 275-285

8. Fernàndez M.S-B., Alegre Calderón J.M., Bravo Diez P.M., Cuesta Segura I.I., 2010, Stress-separation techniques in photoelasticity, Strain, 1, 1-17

9. Frocht M., 1931, A Study of Photoelasticity and a Photoelastic Theoretical Investigation of the Stress Distribution in Square Blocks Subjected to Concentrated Diagonal Loads, Dissertation Topic, Advisor Stephen Prokofyevich Timoshenko. Ph.D. Dissertation, University of Michigan, USA

10. Frocht M., 1941, Photoelasticity, Volume I, John Wiley \& Sons, New York

11. Frocht M., 1948, Photoelasticity, Volume II, John Wiley \& Sons, New York

12. Hetnarski R.B., Ignaczak J., 2011, The Mathematical Theory of Elasticity, Taylor Francis

13. Kuske A., 1959, Introduction of Photoelasticity (in German), Stuttgart, Wissenschaftliche Verlagsgesellschaft

14. Kuske A., 1971, Handbook of Photoelasticity (in German), Stuttgart: Wiss. Verl. Ges.

15. Kuske A., Robertson G., 1974, Photoelastic Stress Analysis, London, New York, Sydney John Wiley \& Sons

16. Madenci E., Guven I., 2005, The Finite Element Method and Applications in Engineering Using ANSYS, Springer

17. Mangal S.K., Ramesh K., 1999, Use of multiple loads to extract continuous isoclinic fringes by phase-shifting, Strain, 35, 15-17

18. McKenney A., Greengard L., Mayo A., 1996, A fast Poisson solver for complex geometries, Journal of Computation Physics, 2, 348-355

19. Musknelishvili N.I., 1975, Some Basic Problems of the Mathematical Theory of Elasticity, 4th Edit., English translation, L. Noordhoff International Publishing, Leyden

20. Petrucci G., 1997, Full-field automatic evaluation of an isoclinic parameter in white light, Experimental Mechanics, 37, 4, 420-426 
21. Pinit P., Umezaki E., 2007, Digitally whole field analysis of isoclinic parameter in photoelasticity by four-step color phase-shifting technique, Optics and Lasers in Engineering, 45, 7, 795-807

22. Ramesh K., 2008, Photoelasticity, Springer Handbook of Experimental Solid Mechanics, Springer Handbook of Experimental Solid Mechanics

23. REzini D., 1984, Randisochromaten als ausreichende Information zur Spannungstrennung und Spannungsermittelung in der Spannungsoptik, Ph.D. Dissertation, TU-Clausthal, FR Germany

24. Sharafutdinov G. Z., 2012, Basic relations of photoelasticity (I. Allerton Press, Ed.), Allerton Press, Inc., 67, 1, 1-4

25. Shortley G. H., Weller R., 1938, The numerical solution of Laplace's equation, Appl. Phys.

26. Siegmann P., Colombo C., Daz-Garrido F., Patterson E., 2011, Determination of the isoclinic map for complex photoelastic fringe patterns, Experimwntal and Applied Mechanics, 6, 79-85, Conf. Proc. of the SEM Series 17

27. Sмiтн G., 1985, Numerical Solution of Partial Differential Equations: Finite Difference Methods, Oxford Ed., Clarendon Press

28. Zhang J., 1998, Fast and high accuracy multigrid solution of the three dimensional Poisson equation, Journal of Computation Physics, 2, 449-461

Manuscript received October 7, 2013; accepted for print April 17, 2015 